\title{
Sphingosine Kinase 1 Regulates the Pulmonary Vascular Immune Response
}

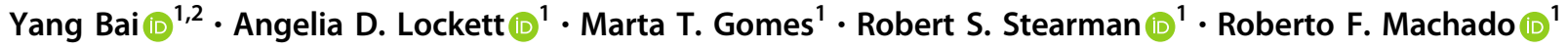

Accepted: 24 May 2021 / Published online: 16 June 2021

(C) The Author(s), under exclusive licence to Springer Science+Business Media, LLC, part of Springer Nature 2021

\begin{abstract}
The aberrant proliferation of pulmonary artery smooth muscle (PASMCs) cells is a defining characteristic of pulmonary arterial hypertension $(\mathrm{PAH})$ and leads to increased vascular resistance, elevated pulmonary pressure, and right heart failure. The sphingosine kinase 1 (SPHK1)/sphingosine-1 phosphate/sphingosine-1 phosphate receptor 2 pathway promotes vascular remodeling and induces PAH. The aim of this study was to identify genes and cellular processes that are modulated by overexpression of SPHK1 in human PASMCs (hPASMCs). RNA was purified and submitted for RNA sequencing to identify differentially expressed genes. Using a corrected p-value threshold of $<0.05$, there were 294 genes significantly up-regulated while 179 were significantly down-regulated. Predicted effects of these differentially expressed genes were evaluated using the freeware tool Enrichr to assess general gene set over-representation (enrichment) and ingenuity pathway analysis $\left(\mathrm{IPA}^{\mathrm{TM}}\right)$ for upstream regulator predictions. We found a strong change in genes that regulated the cellular immune response. IL6, STAT1, and PARP9 were elevated in response to SPHK1 over-expression in hPASMCs. The gene set enrichment mapped to a few immune-modulatory signaling networks, including IFNG. Furthermore, PARP9 and STAT1 protein were elevated in primary hPASMCs isolated from PAH patients. In conclusion, these data suggest a role of Sphk1 regulates pulmonary vascular immune response in PAH.
\end{abstract}

\section{Introduction}

Sphingosine kinase 1 (SPHK1) is a lipid kinase that phosphorylates sphingosine to generate the bioactive sphingolipid sphingosine-1 phosphate (S1P). S1P signals intracellularly or can be secreted and activates intracellular signaling via binding several high-affinity G-protein coupled receptor family members, sphingosine- 1 phosphate receptor (S1PR) 1-5. The SPHK1/S1P/S1PR pathway is a

These authors contributed equally: Yang Bai, Angelia D. Lockett.

Supplementary information The online version contains supplementary material available at https://doi.org/10.1007/s12013021-01006-8.

Roberto F. Machado

robmacha@iu.edu

1 Division of Pulmonary, Critical Care, Sleep and Occupational Medicine, Indiana University School of Medicine, Indianapolis, IN, USA

2 Department of Clinical Pharmacology, School of Pharmacy, China Medical University, Shenyang, Liaoning, China critical regulator of several physiological processes including $\mathrm{Ca}^{2+}$ homeostasis, cell survival, migration, and inflammation [1]. The S1PR system regulates immune cell trafficking through the circulatory and lymphatic systems as well as modulates vascular barrier function to control the extravasation of immune cells $[2,3]$. Under normal conditions, SPHK1/S1P/S1PR is involved in the maintenance of barrier integrity. Conversely, during inflammation, the signaling axis promotes inflammatory signaling by increasing vascular leakiness [4]. Pathological signaling of SPHK1/ S1P/S1PR is involved in multiple diseases among which are cancer, diabetes, atherosclerosis, osteoporosis, and pulmonary arterial hypertension (PAH) [5].

In PAH, SPHK1/S1P induces vascular remodeling by promoting the proliferation of pulmonary artery smooth muscle cells (PASMCs) [6]. Hyperproliferation of PASMCs leads to narrowed pulmonary arteries resulting in increased pulmonary vascular resistance which ultimately results in death due to right heart failure. PAH has multiple etiologies and develops as a co-morbidity of diseases that are characterized by excessive or aberrant inflammatory signaling, such as scleroderma, systemic lupus erythematosus, and human immunodeficiency virus (HIV) infection [7-9]. Notably, experimental PAH can be 
induced in animals by exposure to immune-stimulatory triggers such as HIV and interleukin 6 (IL6) [10, 11]. The available pharmaceutical interventions for PAH target mostly vasoconstriction but fail to address the underlying causes of remodeling of pulmonary vascular cells. Hence, there is an urgent need to identify the molecular mechanisms that promote the abnormal proliferation of PASMCs. Therefore, we over-expressed SPHK1 in human PASMCs and performed RNA sequencing (RNAseq) analysis to identify novel pathways that regulate pulmonary vascular cell function.

\section{Materials and Methods}

\section{Reagents and Antibodies}

Smooth muscle cell growth medium (SMGM2) and supplements were purchased from Lonza (Walkersville, MD). Rabbit anti-signal transducer and activator of transcription 1 (STAT1) and rabbit IgG secondary antibodies were from Cell Signaling Technology (Danvers, MA). Horseradish peroxidase-conjugated $\beta$-actin antibody was obtained from Sigma (St. Louis, MO). RIPA buffer, protease inhibitor cocktail, and Super Signal West Femto were from Thermo Fisher Scientific (Waltham, MA).

\section{Cell Culture and Transfection}

Human pulmonary artery smooth muscle cells (hPASMCs) were obtained from Lonza. IPAH and control patient cells were obtained from the pulmonary hypertension breakthrough initiative (PHBI) Cell Core (Philadelphia, PA). Cells were cultured in SMGM2 containing growth supplements and 5\% FBS (complete medium) at $37{ }^{\circ} \mathrm{C}$ in $5 \% \mathrm{CO}_{2}$ in a humidified tissue culture incubator. Human SPHK1 (Myc-DDK-tagged) plasmid and pCMV6 empty vector were purchased from Ori Gene (Rockville, MD). For transfection, 300,000 hPASMCs were cultured on 6-well plates followed by transfection of $3.75 \mu \mathrm{g}$ of plasmid using Xfect transfection reagent (Takara, Mountain View, CA) according to the manufacturer's protocol. All experiments were performed in low passage cells $(\leq 8)$. Cells were transfected in a complete medium. The medium was replaced with a complete medium after $4 \mathrm{~h}$, and the cells were collected after $48 \mathrm{~h}$ and stored at $-80^{\circ} \mathrm{C}$.

\section{RNAseq and Pathway Analysis}

Total RNA was purified from transfected hPASMCs overexpressing SPHK1, as well as transfected vector DNA control, by using the RNeasy Plus Mini Kit (RNA >200 nucleotides: Qiagen, Germantown, MD). Three cell replicates were harvested for either the vector control of SPHK1 over-expression.
The average RIN for the RNA was 9.4, an indication of good quality preparations. Total RNA was submitted to the Indiana School of Medicine Center for Medical Genomics (Indianapolis, IN) for RNAseq (Illumina) and differentially expressed gene list generation. In brief, the Genomics Core used the KAPA mRNA Hyperprep Kit for library preparation, running on a NovaSeq6000 for 100 base-paired end reads. The Core's standard RNAseq quantitation work-follow consisted of accessing sequence quality (FastQC and MultiQC (https://www.bioinformatics.babraham.ac.uk/projects/fastqc/ and https://multiqc.info/)), alignment to UCSC hg38 human genome (STAR aligner [12]), mapping quality (NGSUtils, bamutils [13]), read counting (subread/feature Counts [14]), and differential expression (DE) analysis (edgeR [15]). An average of $\sim 29$ million reads was uniquely mapped per sample with $\sim 2.3 \%$ rRNA contamination. The .bam files have been deposited at GEO (https://www.ncbi.nlm.nih.gov/geo/) under the accession GSE174304.

For gene set enrichment (GSE) analysis and upstream regulator predictions, Enrichr (https://maayanlab.cloud/ Enrichr/) and ingenuity pathway analysis (IPA ${ }^{\mathrm{TM}}$ ) were used. Enrichr utilizes the DE gene list (gene symbols only) for its multi-database analyses. Enrichr provides a single-webtool location to query a wide variety of databases for GSE patterns. Section 1A (Tissues) and 1D (transcription factor co-expression) are derived from the ARCHS4 compilation (https://maayanlab.cloud/archs4/). Section 1B (gene ontology (GO) biological process (BP)) is derived from the GO website (http://geneontology.org/). Section 1C is compiled by the Enrichr project with the primary data derived from the ENCODE project (https://www.encodeproject.org/) and the ChIP-X project (https://maayanlab.cloud/Harmonizome/resource/ChIP-X + Enrichment + Analysis).

IPA uses both the gene symbols and the $\log _{2}$ (foldchange) expression ratios. This enables IPA to estimate whether an enriched pathway is activated or inhibited through a $z$-score calculation. When a pathway generates a $z$-score $>+2.00$ its evidence for pathway activation, while $z$-score $<-2.00$ indicates pathway inhibition. In addition, zscores are bias-adjusted based on whether a specific DE gene list has a strong non-random bias to it, e.g., a DE gene list composed of interferon-associated genes. In the data presented here, there appears to be a bias toward immune system-associated genes, so when flagged, bias-corrected z-scores are used. The most recent IPA software was used (version 62089861, building coral, updated 02/17/2021).

\section{Experimental Statistical Analysis}

Statistical analysis was performed using Prism 9.0 Software (GraphPad Software, Inc. San Diego, CA). A minimum of three replicates of each experiment was performed and the 
averaged data are expressed as mean \pm SEM. The student's $t$-test was used to compare the differences between the means of two groups and $P<0.05$ was considered statistically significant.

\section{Results}

\section{SPHK1 Over-expression Alters the HPASMC Transcriptome}

HPASMCs over-expressing SPHK1 had a total of 473 differentially regulated genes (294 up; 179 down, Supplementary Table 1) that were significantly differentially expressed (FDR, <0.05; Fig. 1A). Among the up-regulated genes was SPHK1 which was increased by 1.5 -fold (Fig. 1A, B; Supplementary Table 1). Using traditional statistical GSE categorical analyses (as compiled at https://maayanlab.cloud/ Enrichr/ [16]), SPHK1 over-expression modulated expression of genes that regulate multiple cellular processes (Table 1). Enrichr analysis found that the hPASMCs transcriptional pattern after SPHK1 over-expression (GSE adjusted $p$-values $<1.25 \mathrm{E}-14$; Table $1 \mathrm{~A}$ ) was like the $\mathrm{DE}$ patterns of Kupffer, respiratory and vascular smooth muscle cells. This supports a tissue-type shift toward both a vascular adherent and immune cell (Kupffer cells) phenotype while maintaining their original vascular smooth muscle characteristics. The top canonical GO BP was related to interferon- and viral infection responses (GSE adjusted $p$-values $<1.20 \mathrm{E}-15$; Table 1B). Querying the transcription factor ChIP-X database in Enrichr ("X" represents a variety of different genome-wide ChIP-type approaches), two of the top predicted transcription factor binding sites for the DE gene set are interferon-regulatory factor 1 (IRF1) and IRF8 (GSE adjusted $p$-values $<1.01 \mathrm{E}-04$; Table $1 \mathrm{C}$ ). The transcription factor co-expression analysis point to a number of potential immune response regulators (STAT1, STAT2, IRF7, and interferon t-induced protein 16), which when over-expressed, produces a DE pattern similar to SPHK1 over-expression in hPASMCs (GSE adjusted $p$-values $<1.00 \mathrm{E}-25$; Table 1D). This suggests these transcriptional regulators might be potential regulators of the SPHK1 over-expression gene set signature in hPASMCs.

IPA $^{\mathrm{TM}}$ summary of the inter-relationship of DE genes and biological functions is shown in Fig. 2. The most striking feature of this figure is the large set of up-regulated immune response genes (especially in the interferon family) and predicted anti-virus infection state of the hPASMCs. In the diseases and functions analysis, the top categories included replication of virus (GSE adjusted $p$-values $=$ $4.87 \mathrm{E}-18$; predicted to be inhibited, $\mathrm{z}$-score $=-3.73$ ) and antiviral response (GSE adjusted $p$-values $=4.33 \mathrm{E}-20$; predicted to be activated, $z$-score $=+3.23$ ).
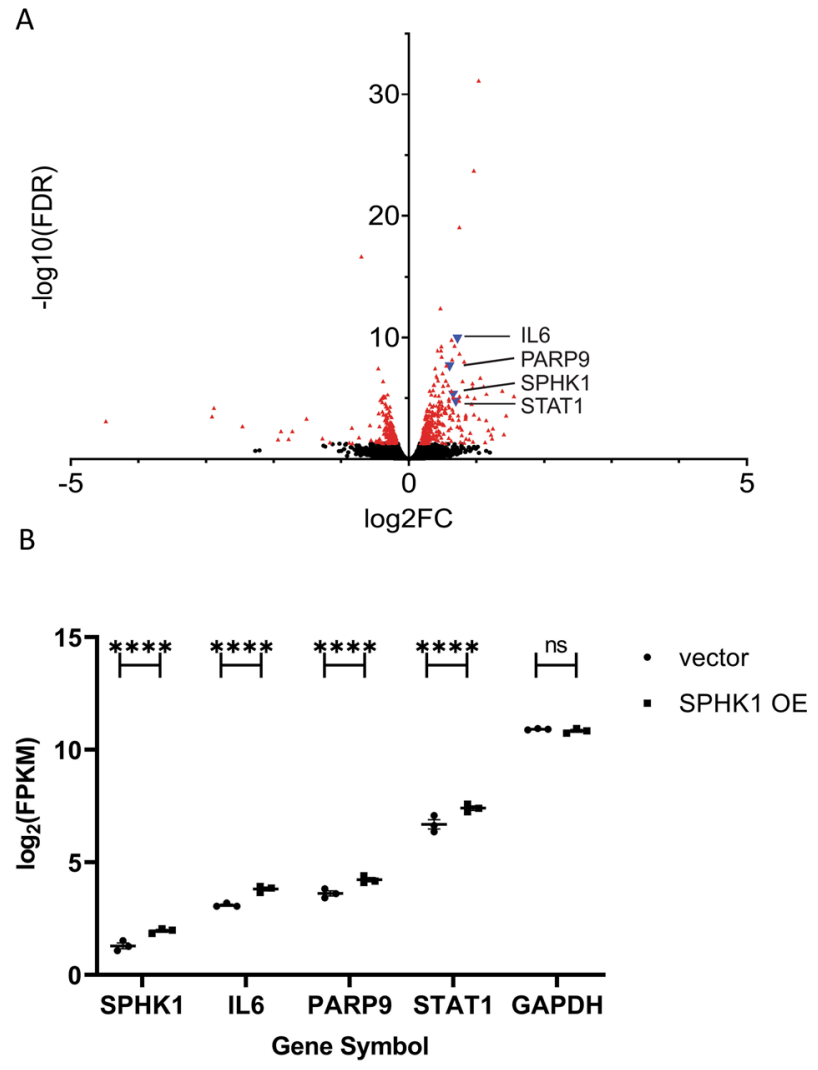

Fig. 1 RNAseq analysis identified 473 significant differentially expressed genes in hPASMCs after SPHK1 over-expression. A A volcano plot $(\log 2$ (FC SPHK1/vector) vs. $-\log 10$ (adjusted $p$-value)) is shown with the significant genes identified in red triangles. The four genes, SPHK1, IL6, PARP9, and STAT1, discussed in detail are identified as blue triangles and labeled with their gene symbols. B The differential expression found by RNAseq analysis (expressed as $\log 2$ (FPKM)) for SPHK1, IL6, PARP9, and STAT1 are shown in ascending expression order. The statistical significance of the DE identified by edgeR analysis is indicated by $* * * *$ (adjusted $p$-value $<$ 1E-04). For comparison, GAPDH, a typical housekeeping gene, was not significantly different $(\mathrm{ns}=$ not significant $)$ and its expression level was $6-7 \times \log 2$-fold higher $(64-128 \times$ fold higher) than STAT1

\section{SPHK1 over-expression leads to an increased cell proliferation gene signature}

Our previous studies demonstrated that SPHK1 overexpression leads to increased cell proliferation and mitogen-activated protein kinase (MAPK) signaling (through extracellular signal-regulated kinase (ERKs, MAPK1/3)) [6]. Though these genes were not specifically found to be up-regulated by increased transcription, the DE pattern suggested that there was activation of this pathway (ERK group GSE adjusted $p$-values $=1.25 \mathrm{E}-8$; predicted to be activated, $z$-score $=+2.3$; Supplementary Table 2). Interestingly, IPA gave differential predictions for MAPK3/ ERK1(p44) vs. MAPK1/ERK2(p42), with MAPK3 predicted to be an activated upstream regulator $(z$-score $=+2.8)$ while MAPK1 was predicted to be an inhibited upstream 
Table 1 Gene set enrichment analysis of differentially expressed genes in hPASMCs after SPHK1 over-expression

\begin{tabular}{|c|c|c|c|}
\hline $\begin{array}{l}\text { A. Enrichr } \\
\text { ARCHS4_Tissues_table }\end{array}$ & Overlap & $\begin{array}{l}\text { Adjusted } \\
P \text {-value }\end{array}$ & Combined Score \\
\hline FIBROBLAST & $151 / 2316$ & $6.58 \mathrm{E}-31$ & 279.0 \\
\hline KUPFFER CELL & $130 / 2316$ & $4.45 \mathrm{E}-20$ & 146.0 \\
\hline $\begin{array}{l}\text { FORESKIN } \\
\text { FIBROBLAST }\end{array}$ & $119 / 2316$ & $2.98 \mathrm{E}-15$ & 98.2 \\
\hline $\begin{array}{l}\text { RESPIRATORY } \\
\text { SMOOTH MUSCLE }\end{array}$ & $118 / 2316$ & $5.95 \mathrm{E}-15$ & 94.5 \\
\hline $\begin{array}{l}\text { VASCULAR } \\
\text { SMOOTH MUSCLE }\end{array}$ & $117 / 2316$ & $1.25 \mathrm{E}-14$ & 90.9 \\
\hline WHARTONS JELLY & $115 / 2316$ & $6.98 \mathrm{E}-14$ & 83.9 \\
\hline CHONDROCYTE & $113 / 2316$ & $3.33 \mathrm{E}-13$ & 77.3 \\
\hline OSTEOBLAST & $113 / 2316$ & $3.33 \mathrm{E}-13$ & 77.3 \\
\hline MYOFIBROBLAST & $108 / 2316$ & $2.02 \mathrm{E}-11$ & 62.5 \\
\hline OMENTUM & $108 / 2316$ & $2.02 \mathrm{E}-11$ & 62.5 \\
\hline \multicolumn{4}{|c|}{ B. Enrichr GO_Biological_Process_2018_table } \\
\hline $\begin{array}{l}\text { Cellular response to type } \\
\text { I interferon } \\
\text { (GO:0071357) }\end{array}$ & $28 / 65$ & $1.50 \mathrm{E}-25$ & 2132.0 \\
\hline $\begin{array}{l}\text { Type I interferon } \\
\text { signaling pathway } \\
\text { (GO:0060337) }\end{array}$ & $28 / 65$ & $1.50 \mathrm{E}-25$ & 2132.0 \\
\hline $\begin{array}{l}\text { SRP-dependent } \\
\text { cotranslational protein } \\
\text { targeting to the membrane } \\
\text { (GO:0006614) }\end{array}$ & $28 / 89$ & $3.23 \mathrm{E}-21$ & 1083.1 \\
\hline $\begin{array}{l}\text { Cotranslational protein } \\
\text { targeting the membrane } \\
(\mathrm{GO}: 0006613)\end{array}$ & $28 / 93$ & $9.57 \mathrm{E}-21$ & 990.4 \\
\hline $\begin{array}{l}\text { Protein targeting to ER } \\
\text { (GO:0045047) }\end{array}$ & $28 / 97$ & $2.81 \mathrm{E}-20$ & 909.7 \\
\hline $\begin{array}{l}\text { Nuclear-transcribed } \\
\text { mRNA catabolic process, } \\
\text { nonsense-mediated decay } \\
\text { (GO:0000184) }\end{array}$ & $29 / 112$ & $1.18 \mathrm{E}-19$ & 759.7 \\
\hline $\begin{array}{l}\text { Viral gene expression } \\
\text { (GO:0019080) }\end{array}$ & $28 / 110$ & $9.00 \mathrm{E}-19$ & 708.2 \\
\hline $\begin{array}{l}\text { Viral transcription } \\
(\mathrm{GO}: 0019083)\end{array}$ & $28 / 113$ & $1.75 \mathrm{E}-18$ & 671.7 \\
\hline $\begin{array}{l}\text { Nnuclear-transcribed } \\
\text { mRNA catabolic process } \\
\text { (GO:0000956) }\end{array}$ & $31 / 174$ & $3.42 \mathrm{E}-16$ & 391.4 \\
\hline $\begin{array}{l}\text { Peptide biosynthetic } \\
\text { process (GO:0043043) }\end{array}$ & $31 / 174$ & $3.42 \mathrm{E}-16$ & 391.4 \\
\hline
\end{tabular}

C. Enrichr ENCODE_and_ChEA_Consensus_TFs_from_ChIP-X_table

\begin{tabular}{lllr}
\hline NELFE ENCODE & $26 / 234$ & $3.38 \mathrm{E}-09$ & 126.6 \\
IRF8 CHEA & $19 / 121$ & $3.38 \mathrm{E}-09$ & 186.8 \\
MYC CHEA & $37 / 573$ & $1.27 \mathrm{E}-06$ & 51.4 \\
KAT2A ENCODE & $12 / 90$ & $3.50 \mathrm{E}-05$ & 87.6 \\
IRF1 ENCODE & $20 / 263$ & $1.01 \mathrm{E}-04$ & 42.8 \\
SOX2 CHEA & $38 / 775$ & $3.30 \mathrm{E}-04$ & 24.2
\end{tabular}


Table 1 (continued)

C. Enrichr ENCODE_and_ChEA_Consensus_TFs_from_ChIP-X_table

\begin{tabular}{|c|c|c|c|}
\hline TAF7 ENCODE & $33 / 640$ & $3.77 \mathrm{E}-04$ & 24.7 \\
\hline MYC ENCODE & $61 / 1515$ & $3.87 \mathrm{E}-04$ & 19.1 \\
\hline RELA ENCODE & $26 / 484$ & $1.09 \mathrm{E}-03$ & 22.4 \\
\hline CEBPD ENCODE & $34 / 734$ & $1.63 \mathrm{E}-03$ & 18.2 \\
\hline \multicolumn{4}{|c|}{ D. Enrichr ARCHS4_TFs_Coexp_table } \\
\hline $\begin{array}{l}\text { STAT1 human tf } \\
\text { ARCHS4 coexpression }\end{array}$ & $66 / 299$ & $1.24 \mathrm{E}-41$ & 1364.5 \\
\hline $\begin{array}{l}\text { STAT2 human tf } \\
\text { ARCHS4 coexpression }\end{array}$ & $62 / 299$ & $1.73 \mathrm{E}-37$ & 1121.9 \\
\hline $\begin{array}{l}\text { ETV7 human tf ARCHS4 } \\
\text { coexpression }\end{array}$ & $59 / 299$ & $1.48 \mathrm{E}-34$ & 961.3 \\
\hline $\begin{array}{l}\text { ZNFX1 human tf } \\
\text { ARCHS4 coexpression }\end{array}$ & $59 / 299$ & $1.48 \mathrm{E}-34$ & 961.3 \\
\hline $\begin{array}{l}\text { PARP12 human tf } \\
\text { ARCHS4 coexpression }\end{array}$ & $57 / 299$ & $1.50 \mathrm{E}-32$ & 863.6 \\
\hline $\begin{array}{l}\text { BATF2 human tf } \\
\text { ARCHS4 coexpression }\end{array}$ & $56 / 299$ & $1.36 \mathrm{E}-31$ & 817.4 \\
\hline $\begin{array}{l}\text { IRF7 human tf ARCHS4 } \\
\text { coexpression }\end{array}$ & $50 / 299$ & $1.01 \mathrm{E}-25$ & 575.6 \\
\hline $\begin{array}{l}\text { IFI16 human tf ARCHS } 4 \\
\text { coexpression }\end{array}$ & $50 / 299$ & $1.01 \mathrm{E}-25$ & 575.6 \\
\hline $\begin{array}{l}\text { TRAFD1 human tf } \\
\text { ARCHS4 coexpression }\end{array}$ & $48 / 299$ & $7.26 \mathrm{E}-24$ & 507.5 \\
\hline $\begin{array}{l}\text { SP100 human tf } \\
\text { ARCHS4 coexpression }\end{array}$ & $43 / 299$ & $2.40 \mathrm{E}-19$ & 361.3 \\
\hline
\end{tabular}

Four selected outputs from Enrichr analysis (https://maayanlab.cloud/Enrichr/) showing the top ten entries, listed by combined score, from each database examined. The overlap value represents how many genes are in the DE gene set divided by the total number of genes grouped into the named annotated gene set. The adjusted $p$-value is based on the Benjamini-Hochberg false discovery rate correction. The combined score is calculated using the unadjusted $p$-value and the $z$-score calculated by a modified Fisher's exact test $(c=\ln (p$-value $) * z$-score $)$. Various benchmarks tested by the Enrichr project suggest the combined score is the best ranking method.

regulator $(z$-score $=-5.3)$. The predicted upstream regulation pattern observed for G1->S progression was also supported by IPA predictions (CCND1 GSE adjusted $p$-values $=1.92 \mathrm{E}-3 ;$ predicted to be activated, $\mathrm{z}$-score $=$ +2.7; cyclin-dependent kinase inhibitor 1A (CDKN1A) GSE adjusted $p$-values $=1.21 \mathrm{E}-6$; predicted to be inhibited, $\mathrm{z}$-score $=-2.2$ ). Several ERK-regulated immediate early genes (FOS, JUN, and EGR1) were predicted to be activated as well (predicted to be activated, $z$-score $>+2.1$ ).

\section{SPHK1 Over-expression Leads to Increased Expression of Genes That Regulate Proinflammatory Signaling Pathways}

Analysis indicated that many of the genes that were upregulated by SPHK1 over-expression are involved in the innate and adaptive immune response (Supplementary Table 1). The poly (ADP-ribose) polymerase 9 (PARP9)-STAT1-IL6 genes were up-regulated by SPHK1 over-expression (Fig. 1B,
PARP9 $(1.5 \times$ up, $q$-value $=2.50 \mathrm{E}-08)$; STAT1 (1.6× up, $q$-value $=2.19 \mathrm{E}-05)$; IL6 $(1.6 \times$ up, $q$-value $=1.33 \mathrm{E}-10)$ ). PARP9 promotes activation of STAT1 downstream of interferon signaling. Several cytokines and chemokines were also up-regulated including IL33, CXCL1 (alias GRO1), CXCL8 (alias IL-8), and CXCL6 (Supplementary Table 1). In addition, members of the tumor necrosis factor ligand superfamily (TNFSF), TNFSF10, and TNFSF13B were significantly increased (Supplementary Table 1). To summarize, SPHK1 over-expression leads to increased expression of many genes that can be upregulated by the interferon-inducible immune response.

\section{Predicted Upstream Regulators of Differential Gene Expression Pattern after SPHK1 Over-expression in hPASMCs}

The top twenty activated or inhibited IPA predicted upstream regulators are shown in Table 2. The complete IPA upstream 
Fig. 2 Ingenuity pathways analysis identifies a strong anti-viral phenotype in hPASMCs after SPHK1 overexpression. The summarized global analysis of the DE gene list in hPASMCs after SPHK1 over-expression suggests a strong anti-viral phenotype. The tightly grouped immune response genes in the center (all up-regulated in the DE gene list) are predicted to down-regulate a wide variety of viral infections (DNA and RNA viruses) though there is an edge effect predicting up-regulation of coronavirus pathogenesis. The IPA legend is included for interpreting the figure

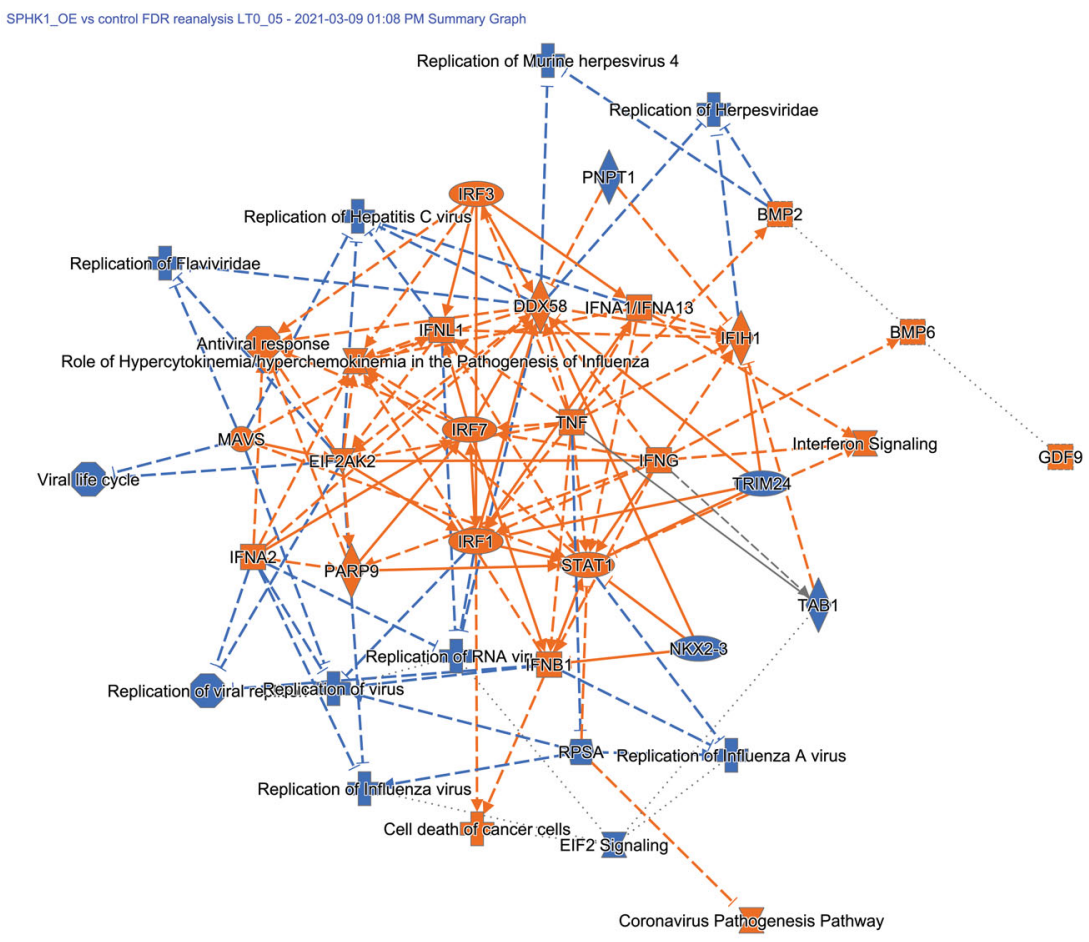

regulators output is included as Supplementary Table 2. As shown above, IL6, STAT1, and PARP9 were both upregulated in hPASMCs after SPHK1 over-expression. In addition, IPA predicted a significant regulatory effect of IL6, STAT1, and PARP9 over-expression predicted from the DE pattern (Figs. 3, 4, and 5 respectively). IL6 and STAT1 can be found in Table 2 showing both a high degree of GSE (the corrected $p$-values $<1.00 \mathrm{E}-15$ ) and strongly predicted activation of their pathways (predicted activated z-scores $>+4.7$ ). In addition, PARP9 is also a predicted upstream regulator (corrected $p$-value $=9.70 \mathrm{E}-10$, predicted activated $z$-score $=$ +3.0, Supplementary Table 2). Thus, IL6, STAT1, and PARP9 are upregulated in hPASMCs over-expressing SPHK1, and the resultant DE gene pattern supports their activating downstream effects as IL6 (cytokine pathway), and PARP9 and STAT1 (transcription regulators).

\section{Global Immune Response Gene Expression Pattern Predicted Downstream Of Interferon-gamma (IFNG) Pathway}

IPA network analysis show that IL6, STAT1, and PARP9 map to the interferon-gamma (IFNG) pathway (Fig. 6). Unlike these genes, IFNG itself is not up-regulated by SPHK1 over-expression. However, the pattern of DE genes strongly supports the activation of IFNG signaling in hPASMCs over-expressing SPHK1 (corrected $p$-value $=3.73 \mathrm{E}-51 ; \mathrm{z}$-score $=+7.7)$. The predicted strong activation is supported by $246 \mathrm{DE}$ genes, from the complete list of 473 , that fall within the IFNG regulation network, and by the direction of gene expression of the vast number of these genes following the expected pattern for IFNG activation. 
Table 2 Ingenuity pathways analysis identified potential upstream regulators

\begin{tabular}{|c|c|c|c|c|c|c|}
\hline Upstream regulator & Expr log-ratio & Molecule type & $\begin{array}{l}\mathrm{B}-\mathrm{H} \text { corrected } \\
p \text {-value }\end{array}$ & $\begin{array}{l}\text { Predicted activation } \\
\text { state }\end{array}$ & $\begin{array}{l}\text { Activation } \\
z \text {-score }\end{array}$ & Flag \\
\hline Lipopolysaccharide & & Chemical drug & $2.91 \mathrm{E}-36$ & Activated & 7.717 & \\
\hline IFNG & & Cytokine & $3.73 \mathrm{E}-51$ & Activated & 7.659 & \\
\hline Poly rI:rC-RNA & & Biologic drug & $4.06 \mathrm{E}-41$ & Activated & 7.474 & \\
\hline IFNA2 & & Cytokine & $5.65 \mathrm{E}-46$ & Activated & 7.396 & \\
\hline TNF & & Cytokine & $8.41 \mathrm{E}-37$ & Activated & 7.240 & \\
\hline IFNL1 & & Cytokine & $2.17 \mathrm{E}-56$ & Activated & 6.871 & \\
\hline LARP1 & & Translation regulator & $4.24 \mathrm{E}-27$ & Activated & 6.844 & Bias \\
\hline Interferon alpha & & Group & $2.50 \mathrm{E}-42$ & Activated & 6.310 & \\
\hline IL1B & & Cytokine & $3.28 \mathrm{E}-28$ & Activated & 6.163 & \\
\hline PRL & & Cytokine & $1.44 \mathrm{E}-45$ & Activated & 5.842 & \\
\hline STAT1 & 0.698 & Transcription regulator & $1.50 \mathrm{E}-31$ & Activated & 5.799 & \\
\hline IRF3 & & Transcription regulator & $7.52 \mathrm{E}-24$ & Activated & 5.694 & \\
\hline IRF1 & & Transcription regulator & $1.22 \mathrm{E}-28$ & Activated & 5.648 & \\
\hline Tretinoin & & $\begin{array}{l}\text { Chemical-endogenous } \\
\text { mammalian }\end{array}$ & $2.42 \mathrm{E}-46$ & Activated & 5.364 & \\
\hline TGM2 & & Enzyme & $1.36 \mathrm{E}-16$ & Activated & 5.294 & \\
\hline Ifnar & & Group & $6.19 \mathrm{E}-20$ & Activated & 5.019 & \\
\hline EIF2AK2 & 0.588 & Kinase & $8.77 \mathrm{E}-21$ & Activated & 4.913 & \\
\hline IRF7 & & Transcription regulator & $4.86 \mathrm{E}-34$ & Activated & 4.903 & Bias \\
\hline IL6 & 0.722 & Cytokine & $1.55 \mathrm{E}-15$ & Activated & 4.730 & \\
\hline IFN beta & & Group & $2.15 \mathrm{E}-26$ & Activated & 4.671 & \\
\hline SOCS1 & & Other & $6.97 \mathrm{E}-11$ & Inhibited & -3.398 & \\
\hline Bisindolylmaleimide I & & Chemical-kinase inhibitor & $5.51 \mathrm{E}-05$ & Inhibited & -3.420 & \\
\hline Dexamethasone & & Chemical drug & $7.81 \mathrm{E}-32$ & Inhibited & -3.429 & \\
\hline 1-Asparaginase & & Biologic drug & $7.01 \mathrm{E}-05$ & Inhibited & -3.450 & \\
\hline BTK & & Kinase & $6.37 \mathrm{E}-08$ & Inhibited & -3.456 & \\
\hline Filgrastim & & Biologic drug & $1.62 \mathrm{E}-12$ & Inhibited & -3.655 & \\
\hline ETV6-RUNX1 & & Fusion gene/product & $2.02 \mathrm{E}-10$ & Inhibited & -3.796 & \\
\hline ACKR2 & & G-protein coupled receptor & $2.42 \mathrm{E}-13$ & Inhibited & -3.873 & \\
\hline SIRT1 & & Transcription regulator & $6.01 \mathrm{E}-11$ & Inhibited & -4.221 & \\
\hline SB203580 & & Chemical-kinase inhibitor & $5.45 \mathrm{E}-16$ & Inhibited & -4.251 & \\
\hline MYC & & Transcription regulator & $8.43 \mathrm{E}-34$ & Inhibited & -4.288 & \\
\hline IRGM & & Enzyme & $1.57 \mathrm{E}-42$ & Inhibited & -4.329 & Bias \\
\hline Irgm1 & & Other & $1.07 \mathrm{E}-31$ & Inhibited & -4.373 & Bias \\
\hline MYCN & & Transcription regulator & $1.59 \mathrm{E}-19$ & Inhibited & -4.568 & \\
\hline $\mathrm{RC} 3 \mathrm{H} 1$ & & Enzyme & $1.81 \mathrm{E}-23$ & Inhibited & -4.600 & \\
\hline MLXIPL & & Transcription regulator & $4.11 \mathrm{E}-17$ & Inhibited & -4.646 & \\
\hline TRIM24 & & Transcription regulator & $3.31 \mathrm{E}-19$ & Inhibited & -5.144 & \\
\hline MAPK1 & & Kinase & $4.91 \mathrm{E}-37$ & Inhibited & -5.265 & \\
\hline IL1RN & & Cytokine & $1.79 \mathrm{E}-30$ & Inhibited & -6.076 & \\
\hline NKX2-3 & & Transcription regulator & $1.73 \mathrm{E}-45$ & Inhibited & -6.641 & \\
\hline
\end{tabular}

The top 40 predicted upstream regulators, based on activation $\mathrm{z}$-score, are listed as the 20 strongest predictions in the activation state (z-score $>$ +2.0 ) or inhibition state (z-score $<-2.0$ ). Upstream regulators can be a wide variety of things including genes (as symbols), compounds, drugs, and pathway groupings. If a predicted upstream regulator is a gene from the DE gene list, the log2(fold-change) is displayed in the Expr Log Ratio column. The molecule type is based on IPA's literature survey. The B-H corrected $p$-value is based on the Benjamini-Hochberg false discovery rate correction. The predicted activation state is based on the activation $z$-score exceeding |2.0|. If the upstream regulator comparison to the DE gene list is predicted to be "bias", a flag is indicated and the activation z-score shown is after bias correction. 
Fig. 3 Ingenuity pathways analysis identifies A network surrounding IL6 overexpression. IL6 was upregulated by SPHK1 overexpression in hPASMCs and IPA built a network of interacting genes, which were also significantly up-regulated (orange/red) or down-regulated (blue/green). The observed pattern of significantly DE genes suggests activation of the IL6 network

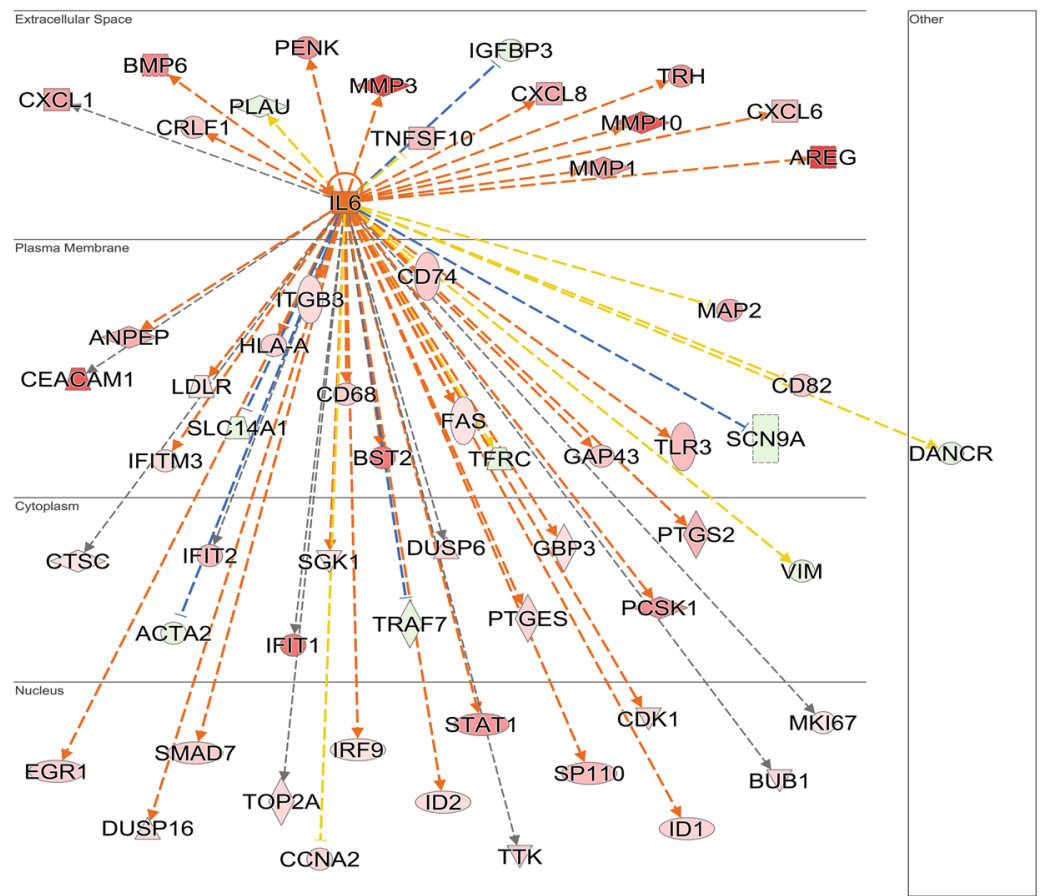

\section{Increased PARP9 and STAT1 in PASMCs Derived from Patients with PAH}

PARP9 and STAT1 protein expression were also increased in PASMCs isolated from idiopathic pulmonary hypertension patients (Fig. 7A, B, $P=0.0007$ and $P<0.0001$, respectively) suggesting a role for this pathway in the pathogenesis of $\mathrm{PAH}$.

\section{Discussion}

We previously described the role of the SPHK $1 / \mathrm{S} 1 \mathrm{P} /$ S1PR2 signaling axis in the development of PAH [6]. SPHK1, S1P, and S1PR2 exhibited increased expression in PASMCs isolated from idiopathic PAH patients. S1P mediates intracellular signaling events in smooth muscle cells via ligating S1PR2. Genetic deletion of SPHK1 or pharmacological inhibition of the S1PR2 prevented hypoxia-induced pulmonary hypertension (HPH) in rodent models. Conversely, partial deletion of S1P lyase (Sphl1), which increases the level of S1P, promoted the development of HPH in vivo. Furthermore, SPHK1 over-expression in hPASMCs leads to increase proliferation in vitro. These studies demonstrate that PASMCs has an essential function in promoting the effects of S1P signaling on PAH development.

In the current study, cellular levels of SPHK1 RNA were increased as expected, due to ectopic expression (Fig. 1). Exposure of PASMCs to S1P has been shown to increase the activation of the ERK (MAPK1/3) pathway leading to cell growth $[6,17]$. We observed divergent predictions from IPA of the upstream regulator status of MAPK3/ERK1 (p44) and MAPK1/ERK2 (p42) after $48 \mathrm{~h}$ of SPHK1 overexpression. ERK1/2 are rapidly and transiently phosphorylated and activated in response to hPASMCs 
Fig. 4 Ingenuity pathways analysis identifies A network surrounding STAT1 overexpression. STAT1 was upregulated by SPHK1 overexpression in hPASMCs and

IPA built a network of interacting genes, which were also significantly up-regulated (orange/red) or down-regulated (blue/green). The observed pattern of significantly DE genes suggests activation of the STAT1 network

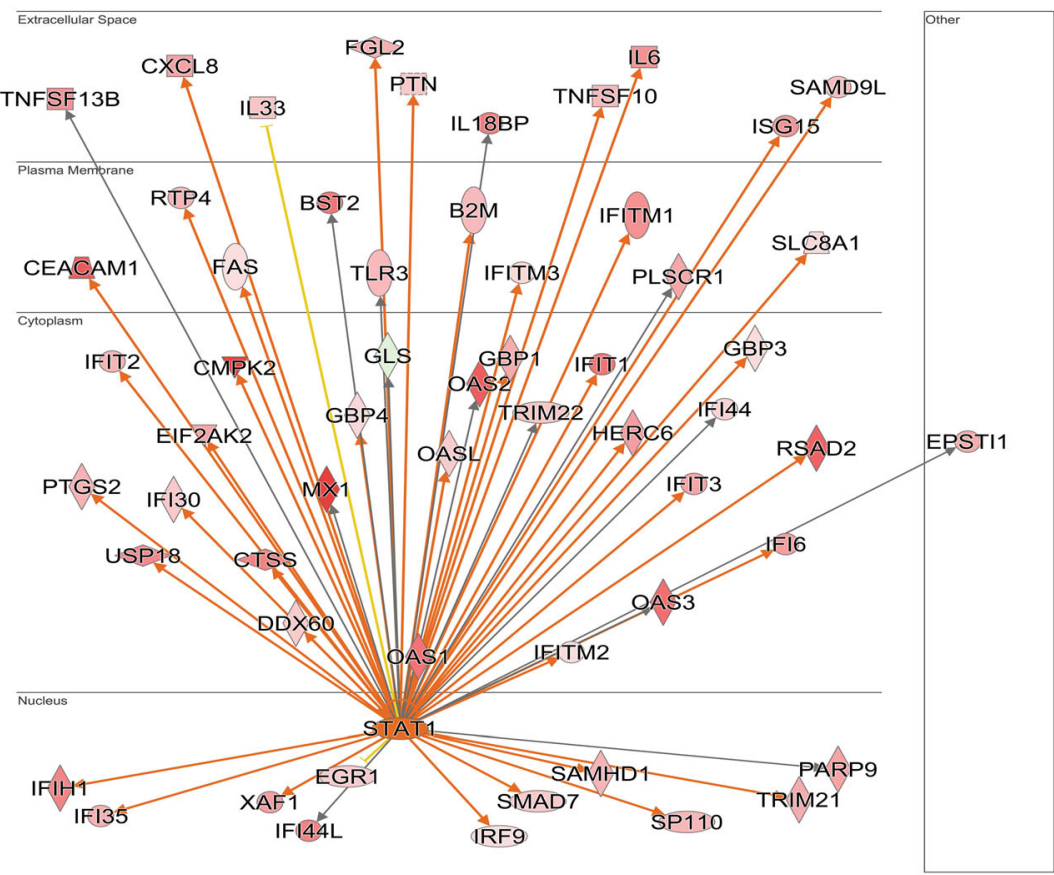

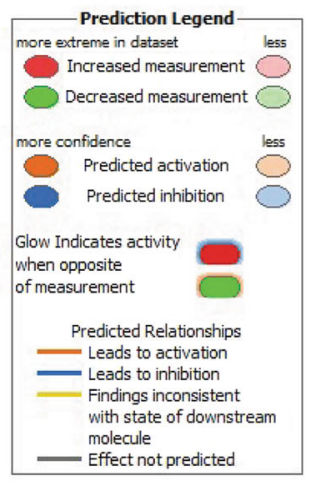

stimulation with S1P. However, the effect of long-term constitutive production of S1P is an area of research that needs deeper exploration. Examining cell cycle regulators showed that SPHK1 over-expression is predicted to promote cell proliferation in $\mathrm{G} 1->\mathrm{S}$ progression by activation of cyclin D1 (CCDN1) and inhibition of CDKN1A.

A preponderance of the genes that were altered by SPHK1 over-expression was involved in different immune regulatory processes. The SPHK/S1P/S1PR signaling axis has an important role in regulating immune processes in that it is known to regulate both immune cell trafficking and vascular integrity [4, 18]. However, much of the current knowledge in this regard is on endothelial cell inflammatory responses. In resting conditions, the S1P gradient between the circulation (high) and in tissues (low) helps to maintain endothelial barrier protection and to control lymphocyte recirculation through the systemic circulation, and lymphoid vasculature and tissue. During activation of inflammatory signaling, the S1P gradient changes, as does expression of S1PR1 leading to increased vascular permeability as well as increased lymphocyte egress and recruitment to sites of inflammation. This process also involves the signaling of pro-inflammatory cytokines and chemokines $[4,18]$.

We found that SPHK1 over-expression significantly increased IL6, PARP9, and STAT1 gene expression in hPASMCs (Fig. 1). In macrophage cells, PARP9 is upstream of STAT1 activation as it was demonstrated that its expression is necessary for IFNG induced STAT1 phosphorylation, regulating the expression of INFG induced pro-inflammatory cytokines and chemokines, tumor necrosis factor $\alpha(\mathrm{TNF} \alpha)$, interleukin-1 $\beta$ (IL-1 $\beta$ ), and chemokine (C-C motif) ligand 2 (CCL2) (alias MCP1) [19]. In hPASMCs, SPHK1-overexpression increased expression of members of the TNF super ligand family (TNFSF10 and TNFSF13B) and of the interleukin family 
Fig. 5 Ingenuity pathways analysis identifies a network surrounding PARP9 overexpression. PARP9 was upregulated by SPHK1 overexpression in hPASMCs and IPA built a network of interacting genes, which were also significantly up-regulated (orange/red). The observed pattern of significantly DE genes suggests activation of the PARP9 network

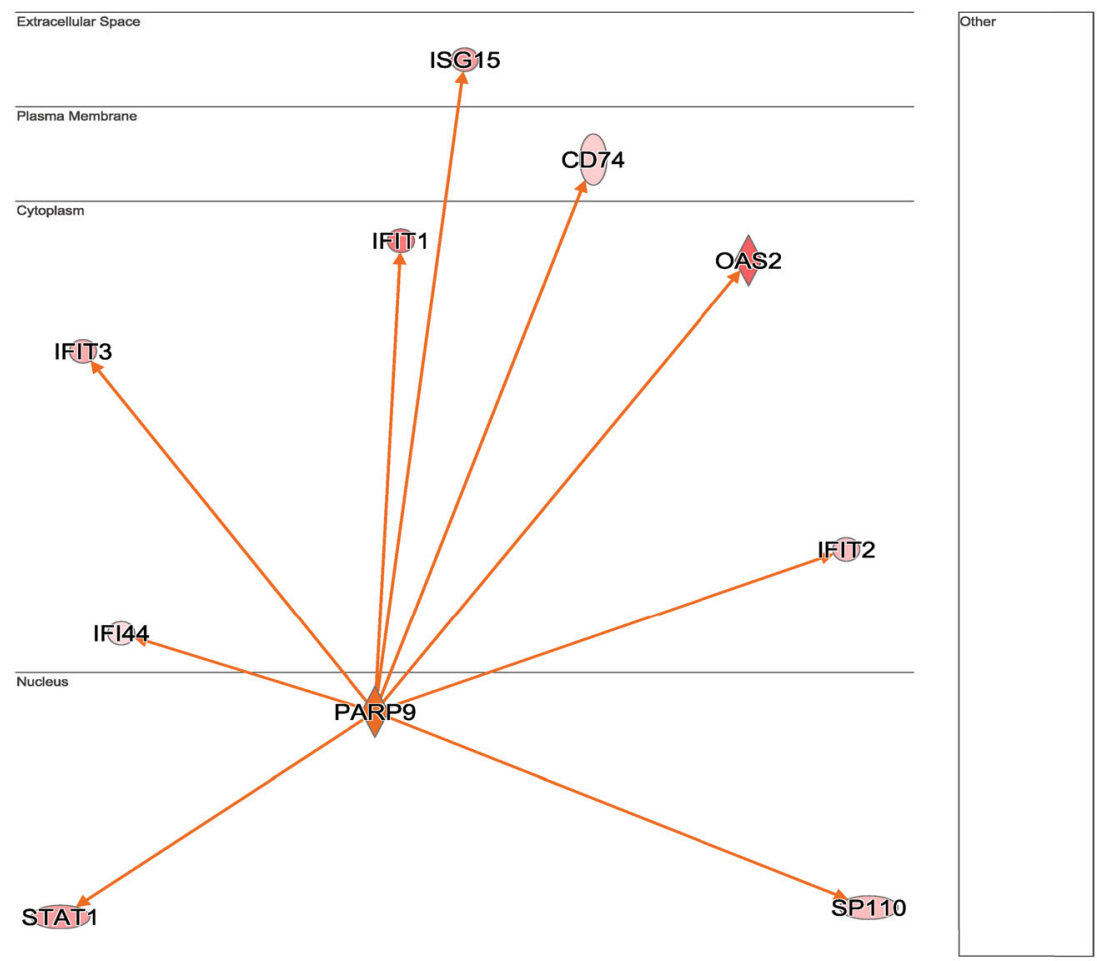

cytokine, IL33. Expression of chemokines, chemokine (C-X-C motif) ligand 8 (CXCL8, IL-8), CXCL1 (GRO1), and CXCL6 were also increased. Toll-like receptor 3 (TLR3) expression was also increased and maps to the IL6 signaling network (Fig. 3). Notably, activation of TLR3 leads to IL6 secretion from bronchial epithelial cells [20]. Circulating levels of IL6 are elevated in PAH patients and correlates with worse right ventricular function [21]. Over-expression of IL6 in animals leads to increased muscularization of the pulmonary vasculature and induces spontaneous pulmonary hypertension that is worsened with hypoxia [11, 22]. Furthermore, smooth muscle-specific knock-out of the interleukin 6 receptor (IL6R), provides protection against HPH [23].

In addition to up-regulating mediators of proinflammatory signaling, SPHK1 over-expression also enhanced the expression of antiviral genes that support host defense against viral replication. Each of these inflammatory mediators is involved in the IFNG signaling network (Fig. 6). The interferon-induced protein with tetratricopeptide repeat (IFIT) family consists of four members, IFIT1, IFIT2, IFIT3, and IFIT5, that bind to viruses and prevent viral replication by modulating both viral and host cell function [24]. SPHK1 over-expression enhanced the expression of each IFIT member as well as other interferonregulated antiviral genes. Among the genes that increased were interferon-induced transmembrane protein 1 (IFITM1) which prevents viral entry to host cells, and interferoninduced protein 44-like (IFI44L) which can prevent viral replication without altering viral binding to host cells [25]. All these interferon enhanced genes were associated with up-regulated STAT1 gene expression when pathway analysis was performed (Fig. 4). Pathway analysis also suggests that the effect of up-regulation of these genes on the 
Fig. 6 Ingenuity pathways analysis predicts INFG is an upstream transcriptional regulator. IFNG is predicted to be an important upstream regulator of the observed $\mathrm{DE}$ gene pattern in hPASMCs after SPHK1 over-expression, though IFNG itself was not observed to be transcriptionally upregulated. IPA built a network of genes potentially stemming from IFNG, which were significantly up-regulated (orange/red) or down-regulated (blue/green) The observed pattern of significantly DE genes suggests activation of the extensive IFNG network
Fig. 7 Idiopathic PAH patients have increased protein levels of PARP9 and STAT1.

Western blot showing that PASMCs isolated from patients have increased PARP9 (A) and STAT1 (B) expression. Quantification and normalization to $\beta$-actin demonstrate a significant increase in patients $(n=5)$ when compared to control subjects $(n=7) . * * * p=.0007$ versus control $* * * * P<0.0001$ versus control
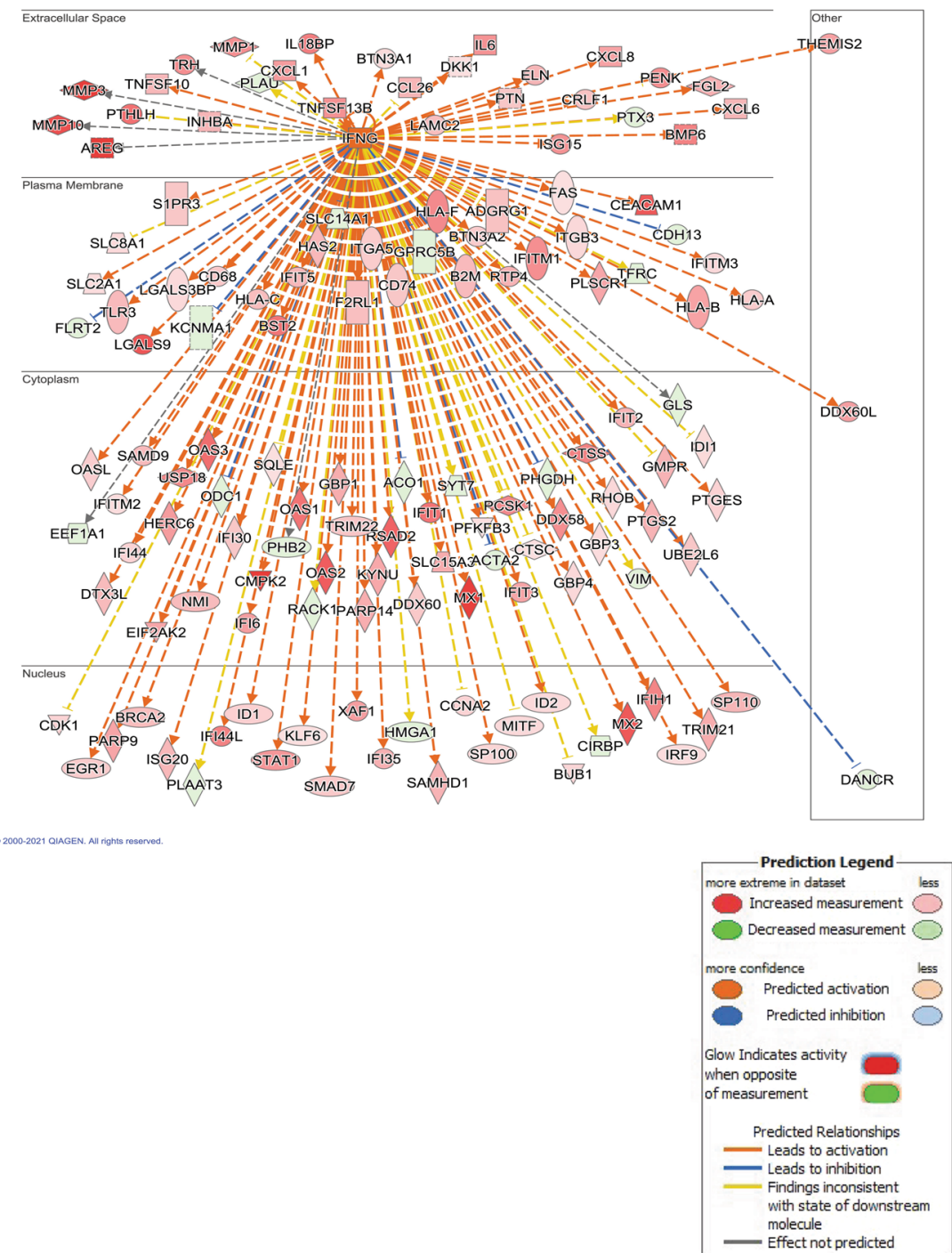

A
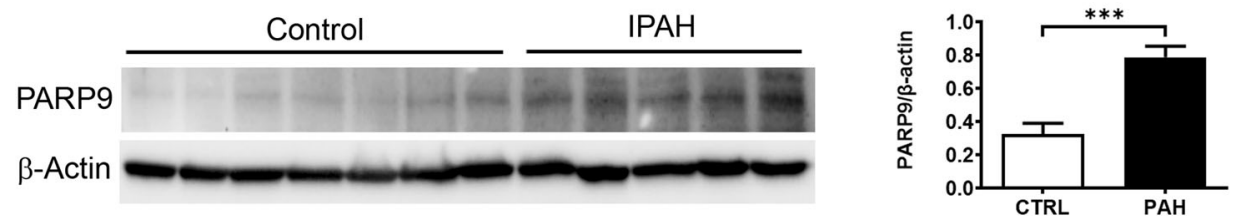

B

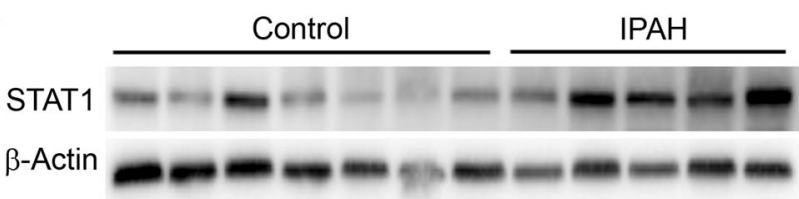

viral host defense is dependent on the type of virus. For example, replication of Herpesvirus, influenza, and hepatitis $\mathrm{C}$ virus was predicted to be inhibited while the Coronavirus pathway was predicted to be activated (Fig. 2). Importantly, anti-SPHK/S1P/S1PR compounds have been suggested as a potential therapeutic approach to treat the SARS-CoV-2 virus (causes COVID-19 disease) as a mechanism to control viral replication [26]. There is a potential link between COVID-19 and pulmonary vascular dysfunction. Patients who succumbed to COVID-19 were found to have 
thickened pulmonary vascular walls, a major hallmark of $\mathrm{PAH}$ [27]. This observation is particularly noteworthy as other viruses, such as HIV, human Herpesvirus-8 (HHV-8) and hepatitis $\mathrm{C}$ are also risk factors for developing PAH $[9,28-31]$.

Dysregulation of the immune response is clearly associated with the pathobiology of PAH [10, 31-39]. The data presented herein suggest that the SPHK1/S1P/S1PR2 signaling axis is important for the regulation of the vascular smooth muscle immune response and highlight the importance of investigating the role of these responses in PAH and other vascular pathologies.

Acknowledgements This work was supported by grants from the National Heart, Lung, and Blood Institute of the National Institutes of Health (R01 HL127342, R01HL111656-R.F.M.) and the National Nature Science Foundation of China (No. 81803530-Y.B.).

Author Contributions Conceptualization: R.F.M.; Methodology: R.F.M., Y.B., A.D.L., R.S.S.; Data collection and analysis: Y.B. and R.S.S.; Writing-first draft: A.D.L.; Writing-review and editing: R.S.S., A.D.L., Y.B., R.F.M.

\section{Compliance with Ethical Standards}

Conflict of Interest The authors declare no competing interests.

Publisher's note Springer Nature remains neutral with regard to jurisdictional claims in published maps and institutional affiliations.

\section{References}

1. Ogretmen, B. \& \& Hannun, Y. A. (2004). Biologically active sphingolipids in cancer pathogenesis and treatment. Nature Reviews Cancer, 4, 604-16.

2. Garcia, J. G. et al. (2001). Sphingosine 1-phosphate promotes endothelial cell barrier integrity by Edg-dependent cytoskeletal rearrangement. Journal of Clinical Investigation, 108, 689-701.

3. Pham, T. H. et al. (2010). Lymphatic endothelial cell sphingosine kinase activity is required for lymphocyte egress and lymphatic patterning. Journal of Experimental Medicine, 207, 17-27.

4. Swan, D. J., Kirby, J. A. \& \& Ali, S. (2010). Vascular biology: the role of sphingosine 1-phosphate in both the resting state and inflammation. Journal of Cellular and Molecular Medicine, 14, 2211-22.

5. Maceyka, M. et al. (2012). Sphingosine-1-phosphate signaling and its role in disease. Trends in Cell Biology, 22, 50-60.

6. Chen, J. et al. (2014). The sphingosine kinase 1/sphingosine-1phosphate pathway in pulmonary arterial hypertension. American Journal of Respiratory and Critical Care Medicine, 190, 1032-43.

7. Chaisson, N. F., \& Hassoun, P. M. (2013). Systemic sclerosisassociated pulmonary arterial hypertension. Chest, 144, 1346-56.

8. Kommireddy, S., et al. (2015). Pulmonary arterial hypertension in systemic lupus erythematosus may benefit by addition of immunosuppression to vasodilator therapy: an observational study. Rheumatology, 54, 1673-9.

9. Tcherakian, C. et al. (2013). Inflammatory mechanisms in HIV-associated pulmonary arterial hypertension. Seminars in Respiratory and Critical Care Medicine, 34, 645-53.

10. George, M. P. et al. (2013). Physiologic changes in a nonhuman primate model of HIV-associated pulmonary arterial hypertension.
American Journal of Respiratory Cell and Molecular Biology, 48, 374-81.

11. Steiner, M. K., et al. (2009). Interleukin-6 overexpression induces pulmonary hypertension. Circulation Research, 104, 236-44.

12. Dobin, A., et al. (2013). STAR: ultrafast universal RNA-seq aligner. Bioinformatics, 29, 15-21.

13. Breese, M. R., \& Liu, Y. (2013). NGSUtils: a software suite for analyzing and manipulating next-generation sequencing datasets. Bioinformatics, 29, 494-6.

14. Liao, Y., Smyth, G. K., \& Shi, W. (2014). featureCounts: an efficient general purpose program for assigning sequence reads to genomic features. Bioinformatics, 30, 923-30.

15. Robinson, M. D., McCarthy, D. J., \& Smyth, G. K. (2010). edgeR: a bioconductor package for differential expression analysis of digital gene expression data. Bioinformatics, 26, 139-40.

16. Xie, Z., et al. (2021). Gene set knowledge discovery with enrichr. Current Protocols, 1, e90.

17. Kimura, T., et al. (2000). Sphingosine 1-phosphate stimulates proliferation and migration of human endothelial cells possibly through the lipid receptors, Edg-1 and Edg-3. Biochemical Journal, 348, 71-6.

18. Obinata, H., \& Hla, T. (2019). Sphingosine 1-phosphate and inflammation. International Immunology, 31, 617-25.

19. Iwata, H., et al. (2016). PARP9 and PARP14 cross-regulate macrophage activation via STAT1 ADP-ribosylation. Nature Communications, 7, 12849.

20. Melkamu, T., Kita, H. \& \& O'Grady, S. M. (2013). TLR3 activation evokes IL-6 secretion, autocrine regulation of Stat 3 signaling and TLR2 expression in human bronchial epithelial cells. Cell Communication and Signaling, 7, 109-18.

21. Prins, K. W., et al. (2018). Interleukin-6 is independently associated with right ventricular function in pulmonary arterial hypertension. Journal of Heart and Lung Transplant, 37, 376-84.

22. Savale, L. et al. (2009). Impact of interleukin-6 on hypoxiainduced pulmonary hypertension and lung inflammation in mice. Respiratory Research, 10, 6.

23. Tamura, Y. et al. (2018). Ectopic upregulation of membranebound IL6R drives vascular remodeling in pulmonary arterial hypertension. Journal of Clinical Investigation, 128, 1956-70.

24. Zhou, X. et al. (2013). Interferon induced IFIT family genes in host antiviral defense. International Journal of Biological Sciences, 9, 200-8.

25. Busse, DC. et al.(2020). Interferon-induced protein 44 and interferon-induced protein 44-like restrict replication of respiratory syncytial virus. Journal of Virology, 94, e00297-20.

26. McGowan, EM. et al.(2020). Targeting the SphK-S1P-SIPR pathway as a potential therapeutic approach for COVID-19. International Journal of Molecular Sciences, 21, 7189.

27. Suzuki, Y. J. et al. (2021). COVID-19 patients may become predisposed to pulmonary arterial hypertension. Medical Hypotheses, 147, 110483.

28. Bull, T. M. et al. (2003). Primary pulmonary hypertension, Castleman's disease and human herpesvirus-8. European Respiratory Journal, 22, 403-7.

29. Chang, Y., et al. (1994). Identification of herpesvirus-like DNA sequences in AIDS-associated Kaposi's sarcoma. Science, 266, 1865-9.

30. Nicastri, E., et al. (2005). Human herpesvirus 8 and pulmonary hypertension. Emerging Infectious Diseases, 11, 1480-2.

31. Demir, C. \& \& Demir, M. (2014). Effect of hepatitis C virus infection on the right ventricular functions, pulmonary arterypressure and pulmonary vascular resistance. International Journal of Clinical and Experimental Medicine, 7, 2314-8.

32. Daley, E. et al. (2008). Pulmonary arterial remodeling induced by a Th2 immune response. Journal of Experimental Medicine, 205, 361-72. 
33. Kumar, R., \& Graham, B. (2018). IL-33-HIF1alpha axis in hypoxic pulmonary hypertension. EBioMedicine, 33, 8-9.

34. Kumar, R \& Graham, B. (2018). How does inflammation contribute to pulmonary hypertension? Eur Respir J. 51, 1702403.

35. Kumar, R. et al. (2015). The causal role of IL-4 and IL-13 in Schistosoma mansoni pulmonary hypertension. American Journal of Respiratory and Critical Care Medicine, 192, 998-1008.

36. Kumar, R. et al. (2020). Interstitial macrophage-derived thrombospondin-1 contributes to hypoxia-induced pulmonary hypertension. Cardiovascular Research, 116, 2021-30.
37. Kumar, R. et al. (2019). Th2 CD4(+) T cells are necessary and sufficient for Schistosoma-pulmonary hypertension. Journal of the American Heart Association, 8, e013111.

38. Rathinasabapathy, A., et al. (2018). The selective angiotensin II type 2 receptor agonist, compound 21, attenuates the progression of lung fibrosis and pulmonary hypertension in an experimental model of bleomycin-induced lung injury. Frontiers in Physiology, 9, 180.

39. Sharma, R. K., et al. (2018). Involvement of neuroinflammation in the pathogenesis of monocrotaline-induced pulmonary hypertension. Hypertension, 71, 1156-63. 